\title{
ON ZYGMUND-TYPE INEQUALITIES CONCERNING POLAR DERIVATIVE OF POLYNOMIALS
}

\author{
Nisar Ahmad Rather \\ University of Kashmir, \\ Hazratbal, Srinagar, Jammu and Kashmir 190006, India \\ dr.narather@gmail.com

\section{Suhail Gulzar} \\ Government College of Engineering and Technology, \\ Safapora, Ganderbal, Jammu and Kashmir 193504, India \\ sgmattoo@gmail.com \\ Aijaz Bhat \\ University of Kashmir, \\ Hazratbal, Srinagar, Jammu and Kashmir 190006, India \\ aijazb824@gmail.com
}

\begin{abstract}
Let $P(z)$ be a polynomial of degree $n$, then concerning the estimate for maximum of $\left|P^{\prime}(z)\right|$ on the unit circle, it was proved by S. Bernstein that $\left\|P^{\prime}\right\|_{\infty} \leq n\|P\|_{\infty}$. Later, Zygmund obtained an $L_{p}$-norm extension of this inequality. The polar derivative $D_{\alpha}[P](z)$ of $P(z)$, with respect to a point $\alpha \in \mathbb{C}$, generalizes the ordinary derivative in the sense that $\lim _{\alpha \rightarrow \infty} D_{\alpha}[P](z) / \alpha=P^{\prime}(z)$. Recently, for polynomials of the form $P(z)=a_{0}+\sum_{j=\mu}^{n} a_{j} z^{j}, 1 \leq \mu \leq n$ and having no zero in $|z|<k$ where $k>1$, the following Zygmund-type inequality for polar derivative of $P(z)$ was obtained:$$
\left\|D_{\alpha}[P]\right\|_{p} \leq n\left(\frac{|\alpha|+k^{\mu}}{\left\|k^{\mu}+z\right\|_{p}}\right)\|P\|_{p}, \quad \text { where } \quad|\alpha| \geq 1, \quad p>0 .
$$

In this paper, we obtained a refinement of this inequality by involving minimum modulus of $|P(z)|$ on $|z|=k$, which also includes improvements of some inequalities, for the derivative of a polynomial with restricted zeros as well.
\end{abstract}

Keywords: $L^{p}$-inequalities, Polar derivative, Polynomials.

\section{Zygmund type inequalities for polynomials}

Let $\mathcal{P}_{n}$ denote the space of all complex polynomials of degree at most $n$. Define

$$
\|P\|_{p}:=\left(\frac{1}{2 \pi} \int_{0}^{2 \pi}\left|P\left(e^{i \theta}\right)\right|^{p} d \theta\right)^{1 / p}, \quad 0<p<\infty
$$

It is well known that the supremum norm satisfies

$$
\|P\|_{\infty}:=\max _{|z|=1}|P(z)|=\lim _{p \rightarrow \infty}\|P\|_{p} .
$$


It is also known [11] that $\lim _{p \rightarrow 0}\|P\|_{p}=\|P\|_{0}$, where

$$
\|P\|_{0}:=\exp \left(\frac{1}{2 \pi} \int_{0}^{2 \pi} \log \left|P\left(e^{i \theta}\right)\right| d \theta\right) .
$$

Let $D_{\alpha}[P](z)$ denote the polar differentiation (see [12]) of a polynomial $P(z)$ of degree $n$ with respect to a complex number $\alpha$, then

$$
D_{\alpha}[P](z):=n P(z)+(\alpha-z) P^{\prime}(z) .
$$

Note that $D_{\alpha}[P](z)$ is a polynomial of degree at most $n-1$ and it generalizes the ordinary derivative $P^{\prime}(z)$ of $P(z)$ in the sense that

$$
\lim _{\alpha \rightarrow \infty} \frac{D_{\alpha}[P](z)}{\alpha}=P^{\prime}(z)
$$

uniformly with respect to $z$ for $|z| \leq R, R>0$.

If $P \in \mathcal{P}_{n}$, then

$$
\left\|P^{\prime}\right\|_{p} \leq n\|P\|_{p} .
$$

Inequality (1.1) is due to Zygmund [21] for the case $p \geq 1$. In its proof, he uses M. Riesz's interpolation formula by means of Minkowski's inequality and obtained this inequality as an $L_{p}$-norm analogue of Bernstein's inequality (for details see [13] or [20]). A natural question was raised here: whether the restriction on $p$ was indeed necessary? The question remained open for quite a long time despite some partial answers. Finally, it was Arestov [1] came up with some remarkable results which among other things proved that the inequality (1.1) remains valid for $0<p<1$ as well. This result is sharp as shown by $P(z)=a z^{n}, a \neq 0$. Arestov [2] also obtained some sharp Bernstein-Zygmund type inequalities for the Szegö composition operators on the set of algebraic polynomials with restrictions on the location of their zeros.

For the class of polynomials $P \in \mathcal{P}_{n}$ having no zero in $|z|<1$, inequality (1.1) can be sharpened. In fact, if $P \in \mathcal{P}_{n}$ and $P(z) \neq 0$ for $|z|<1$, then

$$
\left\|P^{\prime}\right\|_{p} \leq \frac{n}{\|1+z\|_{p}}\|P\|_{p}, \quad p \geq 1
$$

Inequality (1.2) is due to De Bruijn [7]. Later Rahman and Schmeisser [16] followed Arestov's technique and proved that this inequality remains true for $0<p<1$ as well. The estimates is sharp and equality in (1.2) holds for $P(z)=a z^{n}+b,|a|=|b| \neq 0$.

Govil and Rahman [10] generalized inequality (1.2) and proved that if $P \in \mathcal{P}_{n}$ does not vanish in $|z|<k$ where $k \geq 1$, then

$$
\left\|P^{\prime}\right\|_{p} \leq \frac{n}{\|k+z\|_{p}}\|P\|_{p}, \quad p \geq 1
$$

Let $\mathcal{P}_{n, \mu} \subset \mathcal{P}_{n}$ be a class of lacunary type polynomials

$$
P(z)=a_{0}+\sum_{j=\mu}^{n} a_{j} z^{j},
$$

where $1 \leq \mu \leq n$.

As a generalization of inequality (1.3), it was shown by Gardner \& Weems [8] that if $P \in \mathcal{P}_{n, \mu}$ and $P(z) \neq 0$ for $|z|<k, k \geq 1$, then

$$
\left\|P^{\prime}\right\|_{p} \leq \frac{n}{\left\|k^{\mu}+z\right\|_{p}}\|P\|_{p}, \quad p>0 .
$$


Aziz and Rather [5] extended inequality (1.2) to the polar derivative of a polynomial and proved that if $P \in \mathcal{P}_{n}$ and $P(z)$ does not vanish in $|z|<1$, then for $\alpha \in \mathbb{C}$ with $|\alpha| \geq 1$, and $p \geq 1$,

$$
\left\|D_{\alpha}[P]\right\|_{p} \leq n\left(\frac{|\alpha|+1}{\|1+z\|_{p}}\right)\|P\|_{p} .
$$

Concerning the concept and properties of the polar derivative refer to [14].

Aziz et. al [6] also obtained an analogue of inequality (1.3) to the polar derivative and proved that if $P \in \mathcal{P}_{n}$ and $P(z) \neq 0$ for $|z|<k$ where $k \geq 1$, then for $\alpha \in \mathbb{C}$ with $|\alpha| \geq 1$ and $p \geq 1$,

$$
\left\|D_{\alpha}[P]\right\|_{p} \leq n\left(\frac{|\alpha|+k}{\|k+z\|_{p}}\right)\|P\|_{p} .
$$

Rather [17, 18] showed that inequalities (1.5) and (1.6) remain valid for $0<p<1$ as well.

Recently, as a generalization of inequality (1.6), Rather et. al [19] proved that if $P \in \mathcal{P}_{n, \mu}$ and $P(z)$ does not vanish in $|z|<k$ where $k \geq 1$, then for $\alpha \in \mathbb{C}$ with $|\alpha| \geq 1$ and $0 \leq p<\infty$,

$$
\left\|D_{\alpha}[P]\right\|_{p} \leq n\left(\frac{|\alpha|+k^{\mu}}{\left\|k^{\mu}+z\right\|_{p}}\right)\|P\|_{p} .
$$

\section{Main results}

In this paper, we obtain a refinement of inequality (1.7) by involving the minimum modulus of a polynomial. We prove the following main result.

Theorem 1. If $P \in \mathcal{P}_{n, \mu}$ and $P(z)$ does not vanish in $|z|<k$ where $k \geq 1$, then for $\alpha \in \mathbb{C}$ with $|\alpha| \geq 1,0 \leq p \leq \infty$ and $0 \leq t \leq 1$,

$$
\left\|\left|D_{\alpha}[P]\right|+n m t\left(\frac{|\alpha|-1}{1+k^{\mu}}\right)\right\|_{p} \leq n\left(\frac{|\alpha|+k^{\mu}}{\left\|z+k^{\mu}\right\|_{p}}\right)\|P\|_{p}
$$

where $m=\min _{|z|=k}|P(z)|$.

Since

$$
\frac{n m t(|\alpha|-1)}{1+k^{\mu}} \geq 0 \quad \text { for } \quad|\alpha| \geq 1
$$

then one can easily observe that

$$
\left\|D_{\alpha}[P]\right\|_{p} \leq\left\|\left|D_{\alpha}[P]\right|+n m t\left(\frac{|\alpha|-1}{1+k^{\mu}}\right)\right\|_{p},
$$

and this implies that the Theorem 1 is a refinement of inequality (1.7).

If we divide both sides of inequality (2.1) by $|\alpha|$ and let $|\alpha| \rightarrow \infty$, we obtain the following refinement of inequality (1.4).

Corollary 1. If $P \in \mathcal{P}_{n, \mu}$ and $P(z)$ does not vanish in $|z|<k$ where $k \geq 1$, then for $0 \leq p \leq \infty$,

$$
\left\|\left|P^{\prime}\right|+\frac{n m t}{1+k^{\mu}}\right\|_{p} \leq \frac{n}{\left\|z+k^{\mu}\right\|_{p}}\|P\|_{p}
$$

where $m=\min _{|z|=k}|P(z)|$. The result is best possible as shown by the polynomial

$$
P(z)=\left(z^{\mu}+k^{\mu}\right)^{n / \mu},
$$

where $\mu$ divides $n$. 
Inequality (2.2) also includes a refinement of (1.3). By taking $k=1$ and $\mu=1$ in (2.2), the following improvement of inequality (1.2) follows immediately.

Corollary 2. If $P \in \mathcal{P}_{n}$ and $P(z)$ does not vanish in $|z|<1$ then for $0 \leq p \leq \infty$,

$$
\left\|\left|P^{\prime}\right|+\frac{n m t}{2}\right\|_{p} \leq \frac{n}{\|1+z\|_{p}}\|P\|_{p},
$$

where $m=\min _{|z|=1}|P(z)|$. The result is sharp and equality in (2.3) holds for $P(z)=z^{n}+1$.

\section{Lemmas}

For the proof of above theorem, we need the following lemmas.

Lemma 1. If

$$
P(z)=a_{0}+\sum_{j=\mu}^{n} a_{j} z^{j}, \quad 1 \leq \mu \leq n,
$$

is a polynomial of degree $n$ having no zeros in $|z|<k$, where $k \geq 1$, then

$$
k^{\mu}\left|P^{\prime}(z)\right| \leq\left|Q^{\prime}(z)\right| \quad \text { for } \quad|z|=1,
$$

where $Q(z)=z^{n} \overline{P(1 / \bar{z})}$.

The above Lemma 1 is implicit in Qazi [15] and the proof of next lemma is implicit in [9].

Lemma 2. If $P(z)$ is a polynomial of degree $n$ having no zero in $|z|<k, k \geq 1$, then for every $\lambda \in \mathbb{C}$ with $|\lambda|<1$,

$$
\left|Q^{\prime}(z)\right| \geq|\lambda| m n \quad \text { for } \quad|z|=1
$$

where

$$
m=\min _{|z|=k}|P(z)|, \quad Q(z)=z^{n} \overline{P(1 / \bar{z})} .
$$

Lemma 3. If

$$
P(z)=a_{0}+\sum_{j=\mu}^{n} a_{j} z^{j}, \quad 1 \leq \mu \leq n,
$$

is a polynomial of degree $n$ having no zeros in $|z|<k$, where $k \geq 1$, then for $0 \leq t \leq 1$,

$$
k^{\mu}\left|P^{\prime}(z)\right| \leq\left|Q^{\prime}(z)\right|-m n t \quad \text { for } \quad|z|=1
$$

where

$$
Q(z)=z^{n} \overline{P(1 / \bar{z})}, \quad m=\min _{|z|=k}|P(z)| .
$$

$\mathrm{P}$ r o o f. By hypothesis, the polynomial $P(z)$ has no zero in $|z|<k, k \geq 1$. We first show for a given $\lambda \in \mathbb{C}$ with $|\lambda|<1$, the polynomial $F(z)=P(z)-\lambda m$ does not vanish in $|z|<k$. This is clear if $m=0$, that is if $P(z)$ has a zero on $|z|=k$. We now suppose that all the zeros of $P(z)$ lie in $|z|>k$, then clearly $m>0$ so that $m / P(z)$ is analytic in $|z| \leq k$ and

$$
\left|\frac{m}{P(z)}\right| \leq 1 \text { for }|z|=k \text {. }
$$


Since $m / P(z)$ is not a constant, by the Minimum modulus principle, it follows that

$$
m<|P(z)| \text { for }|z|<k .
$$

Now, if $F(z)=P(z)-\lambda m$ has a zero in $|z|<k$, say at $z=z_{0}$ with $\left|z_{0}\right|<k$, then

$$
P\left(z_{0}\right)-\lambda m=0 .
$$

This gives

$$
\left|P\left(z_{0}\right)\right|=|\lambda m|=|\lambda| m \leq m, \quad \text { where } \quad\left|z_{0}\right|<k,
$$

which contradicts (3.2). Hence, we conclude that in any case, the polynomial

$$
F(z)=P(z)-\lambda m
$$

does not vanish in $|z|<k, k \geq 1$, for every $\lambda \in \mathbb{C}$ with $|\lambda| \leq 1$. Applying Lemma 1 to

$$
F(z)=P(z)-\lambda m
$$

we get

$$
\left|Q^{\prime}(z)-\bar{\lambda} m n z^{n-1}\right| \geq k^{\mu}\left|P^{\prime}(z)\right| \text { for }|z|=1 .
$$

Now choosing the argument of $\lambda$ so that on $|z|=1$,

$$
\left|Q^{\prime}(z)-\bar{\lambda} m n z^{n-1}\right|=\left|Q^{\prime}(z)\right|-|\lambda| m n
$$

which is possible due to lemma 2. By combining (3.3) and (3.4), we obtain

$$
\left|Q^{\prime}(z)\right| \geq k^{\mu}\left|P^{\prime}(z)\right|+t m n \text { for }|z|=1,
$$

where $t=|\lambda|$ and $0 \leq t<1$. For the case $t=1$, the inequality (3.1) follows immediately by letting $t \rightarrow 1$ in (3.5) and this completes the proof.

The following lemma is due to Aziz and Rather [3].

Lemma 4. If $A, B$ and $C$ are non-negative real numbers such that $B+C \leq A$, then for every real number $\beta$,

$$
\left|(A-C)+e^{i \beta}(B+C)\right| \leq\left|A+e^{i \beta} B\right| .
$$

Lemma 5 [19]. If $a, b$ are any two positive real numbers such that $a \geq b c$ where $c \geq 1$, then for any $x \geq 1, p>0$ and $0 \leq \beta<2 \pi$,

$$
(a+b x)^{p} \int_{0}^{2 \pi}\left|c+e^{i \beta}\right|^{p} d \beta \leq(c+x)^{p} \int_{0}^{2 \pi}\left|a+b e^{i \beta}\right|^{p} d \beta .
$$

We also need the following lemma due to Aziz and Rather [4].

Lemma 6 [4]. If $P \in \mathcal{P}_{n}$ and $Q(z)=z^{n} \overline{P(1 / \bar{z})}$, then for every $p>0$ and $\beta$ real, $0 \leq \beta<2 \pi$,

$$
\int_{0}^{2 \pi} \int_{0}^{2 \pi}\left|P^{\prime}\left(e^{i \theta}\right)+e^{i \beta} Q^{\prime}\left(e^{i \theta}\right)\right|^{p} d \theta d \beta \leq 2 \pi n^{p} \int_{0}^{2 \pi}\left|P\left(e^{i \theta}\right)\right|^{p} d \theta
$$




\section{Proof of Theorem 1}

P r o o f. By hypothesis $P \in \mathcal{P}_{n, \mu}$ and does not vanish in $|z|<k$, where $k \geq 1$ further if

$$
Q(z)=z^{n} \overline{P(1 / \bar{z})},
$$

then, by Lemma 3, we have for $|z|=1$,

$$
k^{\mu}\left|P^{\prime}(z)\right| \leq\left|Q^{\prime}(z)\right|-m n t=\left|Q^{\prime}(z)\right|-m n t\left(\frac{1+k^{\mu}}{1+k^{\mu}}\right) .
$$

Equivalently,

$$
k^{\mu}\left(\left|P^{\prime}(z)\right|+\frac{m n t}{1+k^{\mu}}\right) \leq\left|Q^{\prime}(z)\right|-\frac{m n t}{1+k^{\mu}} \quad \text { for } \quad|z|=1
$$

Setting

$$
A=\left|Q^{\prime}\left(e^{i \theta}\right)\right|, \quad B=\left|P^{\prime}\left(e^{i \theta}\right)\right|, \quad C=\frac{m n t}{1+k^{\mu}}
$$

in Lemma 4 we note by (4.1) that

$$
B+C \leq k^{\mu}(B+C) \leq A-C \leq A, \quad \text { since } \quad k \geq 1 .
$$

Therefore, by Lemma 4 for each real $\beta$, we get

$$
\left|\left(\left|Q^{\prime}\left(e^{i \theta}\right)\right|-\frac{m n t}{1+k^{\mu}}\right)+e^{i \beta}\left(\left|P^{\prime}\left(e^{i \theta}\right)\right|+\frac{m n t}{1+k^{\mu}}\right)\right| \leq|| Q^{\prime}\left(e^{i \theta}\right)\left|+e^{i \beta}\right| P^{\prime}\left(e^{i \theta}\right)|| .
$$

This implies for each $p>0$

$$
\int_{0}^{2 \pi}\left|F(\theta)+e^{i \beta} G(\theta)\right|^{p} d \theta \leq \int_{0}^{2 \pi}|| Q^{\prime}\left(e^{i \theta}\right)\left|+e^{i \beta}\right| P^{\prime}\left(e^{i \theta}\right)||^{p} d \theta
$$

where

$$
F(\theta)=\left|Q^{\prime}\left(e^{i \theta}\right)\right|-\frac{m n t}{1+k^{\mu}} \quad \text { and } \quad G(\theta)=\left|P^{\prime}\left(e^{i \theta}\right)\right|+\frac{m n t}{1+k^{\mu}} .
$$

Let $P^{\prime}(\theta)=\left|P^{\prime}(\theta)\right| e^{i \psi}$ and $Q^{\prime}(\theta)=\left|Q^{\prime}(\theta)\right| e^{i \phi}$, then

$$
\begin{gathered}
\int_{0}^{2 \pi}\left|Q^{\prime}\left(e^{i \theta}\right) e^{i \beta}+P^{\prime}\left(e^{i \theta}\right)\right|^{p} d \beta=\int_{0}^{2 \pi}|| Q^{\prime}\left(e^{i \theta}\right)\left|e^{i(\beta+\phi)}+e^{i \psi}\right| P^{\prime}\left(e^{i \theta}\right)||^{p} d \beta \\
=\int_{0}^{2 \pi}|| Q^{\prime}\left(e^{i \theta}\right)\left|e^{i(\beta+\phi-\psi)}+\right| P^{\prime}\left(e^{i \theta}\right)||^{p} d \beta
\end{gathered}
$$

Putting $\beta+\phi-\psi=\Phi$, then we obtain,

$$
\int_{0}^{2 \pi}\left|Q^{\prime}\left(e^{i \theta}\right) e^{i \beta}+P^{\prime}\left(e^{i \theta}\right)\right|^{p} d \beta=\int_{\phi-\psi}^{2 \pi+\phi-\psi}|| Q^{\prime}\left(e^{i \theta}\right)\left|e^{i \Phi}+\right| P^{\prime}\left(e^{i \theta}\right)||^{p} d \Phi .
$$

Since the function

$$
T(\Phi)=\left|Q^{\prime}\left(e^{i \theta}\right)\right| e^{i \Phi}+\left|P^{\prime}\left(e^{i \theta}\right)\right|
$$


is periodic with period $2 \pi$, hence we have

$$
\begin{aligned}
\int_{0}^{2 \pi} \mid Q^{\prime}\left(e^{i \theta}\right) e^{i \beta} & +\left.P^{\prime}\left(e^{i \theta}\right)\right|^{p} d \beta=\int_{0}^{2 \pi}|| Q^{\prime}\left(e^{i \theta}\right)\left|e^{i \Phi}+\right| P^{\prime}\left(e^{i \theta}\right)||^{p} d \Phi \\
& =\int_{0}^{2 \pi}|| Q^{\prime}\left(e^{i \theta}\right)\left|e^{i \beta}+\right| P^{\prime}\left(e^{i \theta}\right)||^{p} d \beta .
\end{aligned}
$$

Integrating (4.2) both sides with respect to $\beta$ from 0 to $2 \pi$ and using (4.4), we get

$$
\begin{aligned}
\int_{0}^{2 \pi} \int_{0}^{2 \pi}\left|F(\theta)+e^{i \beta} G(\theta)\right|^{p} d \theta d \beta & \leq \int_{0}^{2 \pi} \int_{0}^{2 \pi}|| Q^{\prime}\left(e^{i \theta}\right)\left|+e^{i \beta}\right| P^{\prime}\left(e^{i \theta}\right)||^{p} d \beta d \theta \\
& =\int_{0}^{2 \pi} \int_{0}^{2 \pi}\left|Q^{\prime}\left(e^{i \theta}\right)+e^{i \beta} P^{\prime}\left(e^{i \theta}\right)\right|^{p} d \beta d \theta \\
& =\int_{0}^{2 \pi} \int_{0}^{2 \pi}\left|P^{\prime}\left(e^{i \theta}\right)+e^{i \beta} Q^{\prime}\left(e^{i \theta}\right)\right|^{p} d \theta d \beta
\end{aligned}
$$

By using Lemma 6 this implies,

$$
\int_{0}^{2 \pi} \int_{0}^{2 \pi}\left|F(\theta)+e^{i \beta} G(\theta)\right|^{p} d \theta d \beta \leq 2 \pi n^{p} \int_{0}^{2 \pi}\left|P\left(e^{i \theta}\right)\right|^{p} d \theta .
$$

Now for $|z|=1,0 \leq t \leq 1$ and $\alpha \in \mathbb{C}$ with $|\alpha| \geq 1$ and using the fact that

$$
\left|n P(z)-z P^{\prime}(z)\right|=\left|Q^{\prime}(z)\right|
$$

for $z$ with unit modulus, we have

$$
\begin{aligned}
\left|D_{\alpha}[P]\left(e^{i \theta}\right)\right|+n m t\left(\frac{|\alpha|-1}{1+k^{\mu}}\right) & =\left|n P(z)+(\alpha-z) P^{\prime}(z)\right|+n m t\left(\frac{|\alpha|-1}{1+k^{\mu}}\right) \\
& \leq|\alpha|\left|P^{\prime}(z)\right|+\left|n P(z)-z P^{\prime}(z)\right|+n m t\left(\frac{|\alpha|-1}{1+k^{\mu}}\right) \\
& =|\alpha|\left|P^{\prime}\left(e^{i \theta}\right)\right|+\left|Q^{\prime}\left(e^{i \theta}\right)\right|+n m t\left(\frac{|\alpha|-1}{1+k^{\mu}}\right) \\
& =|\alpha|\left(\left|P^{\prime}\left(e^{i \theta}\right)\right|+\frac{m n t}{1+k^{\mu}}\right)+\left(\left|Q^{\prime}\left(e^{i \theta}\right)\right|-\frac{m n t}{1+k^{\mu}}\right) .
\end{aligned}
$$

By integrating both sides with respect to $\theta$ from 0 to $2 \pi$, for each $p>0$, we get

$$
\begin{gathered}
\int_{0}^{2 \pi}|| D_{\alpha}[P]\left(e^{i \theta}\right)\left|+n m t\left(\frac{|\alpha|-1}{1+k^{\mu}}\right)\right|^{p} d \theta \\
\leq \int_{0}^{2 \pi}|| \alpha\left|\left(\left|P^{\prime}\left(e^{i \theta}\right)\right|+\frac{m n t}{1+k^{\mu}}\right)+\left(\left|Q^{\prime}\left(e^{i \theta}\right)\right|-\frac{m n t}{1+k^{\mu}}\right)\right|^{p} d \theta .
\end{gathered}
$$

Multiply both sides by

$$
\int_{0}^{2 \pi}\left|k^{\mu}+e^{i \beta}\right|^{p} d \beta
$$

we obtain

$$
\begin{gathered}
\int_{0}^{2 \pi}\left|k^{\mu}+e^{i \beta}\right|^{p} d \beta \int_{0}^{2 \pi}|| D_{\alpha}[P]\left(e^{i \theta}\right)\left|+n m t\left(\frac{|\alpha|-1}{1+k^{\mu}}\right)\right|^{p} d \theta \\
\leq \int_{0}^{2 \pi}|| \alpha\left|\left(\left|P^{\prime}\left(e^{i \theta}\right)\right|+\frac{m n t}{1+k^{\mu}}\right)+\left(\left|Q^{\prime}\left(e^{i \theta}\right)\right|-\frac{m n t}{1+k^{\mu}}\right)\right|^{p} d \theta \int_{0}^{2 \pi}\left|k^{\mu}+e^{i \beta}\right|^{p} d \beta .
\end{gathered}
$$


Further, since $k^{\mu} \geq 1,1 \leq \mu \leq n$, and if

$$
a=\left|Q^{\prime}\left(e^{i \theta}\right)\right|-\frac{m n t}{1+k^{\mu}}, \quad b=\left|P^{\prime}\left(e^{i \theta}\right)\right|+\frac{m n t}{1+k^{\mu}}, \quad c=k^{\mu}, \quad x=|\alpha|,
$$

then from (4.1) one can observe that $a \geq b c$. Using Lemma 5 , we get for every $\alpha \in \mathbb{C}$ with $|\alpha| \geq 1$,

$$
\begin{aligned}
& \left\{\left(\left|Q^{\prime}\left(e^{i \theta}\right)\right|-\frac{m n t}{1+k^{\mu}}\right)+|\alpha|\left(\left|P^{\prime}\left(e^{i \theta}\right)\right|+\frac{m n t}{1+k^{\mu}}\right)\right\}^{p} \int_{0}^{2 \pi}\left|k^{\mu}+e^{i \beta}\right|^{p} d \beta \\
\leq & \left(|\alpha|+k^{\mu}\right)^{p} \int_{0}^{2 \pi}\left|\left(\left|Q^{\prime}\left(e^{i \theta}\right)\right|-\frac{m n t}{1+k^{\mu}}\right)+e^{i \beta}\left(\left|P^{\prime}\left(e^{i \theta}\right)\right|+\frac{m n t}{1+k^{\mu}}\right)\right|^{p} d \beta .
\end{aligned}
$$

Again, integrating both sides with respect to $\theta$ from 0 to $2 \pi$, we obtain

$$
\begin{gathered}
\int_{0}^{2 \pi}\left|\left(\left|Q^{\prime}\left(e^{i \theta}\right)\right|-\frac{m n t}{1+k^{\mu}}\right)+\right| \alpha\left|\left(\left|P^{\prime}\left(e^{i \theta}\right)\right|+\frac{m n t}{1+k^{\mu}}\right)\right|^{p} d \theta \int_{0}^{2 \pi}\left|k^{\mu}+e^{i \beta}\right|{ }^{p} d \beta \\
\leq\left(|\alpha|+k^{\mu}\right)^{p} \int_{0}^{2 \pi} \int_{0}^{2 \pi}\left|F(\theta)+e^{i \beta} G(\theta)\right|^{p} d \beta d \theta,
\end{gathered}
$$

where $F(\theta)$ and $G(\theta)$ are given by (4.3). Using this in inequality (4.6), we get

$$
\begin{gathered}
\int_{0}^{2 \pi}\left|k^{\mu}+e^{i \beta}\right| d \beta \int_{0}^{2 \pi}|| D_{\alpha}[P]\left(e^{i \theta}\right)\left|+n m t\left(\frac{|\alpha|-1}{1+k^{\mu}}\right)\right|^{p} d \theta \\
\leq\left(|\alpha|+k^{\mu}\right)^{p} \int_{0}^{2 \pi} \int_{0}^{2 \pi}\left|F(\theta)+e^{i \beta} G(\theta)\right|^{p} d \beta d \theta .
\end{gathered}
$$

By using (4.5) in (4.7), we obtain for each $p>0$ and $|\alpha| \geq 1$

$$
\int_{0}^{2 \pi}\left|k^{\mu}+e^{i \beta}\right| d \beta \int_{0}^{2 \pi}|| D_{\alpha}[P]\left(e^{i \theta}\right)\left|+n m t\left(\frac{|\alpha|-1}{1+k^{\mu}}\right)\right|^{p} d \theta \leq\left(|\alpha|+k^{\mu}\right)^{p} 2 \pi n^{p} \int_{0}^{2 \pi}\left|P\left(e^{i \theta}\right)\right|^{p} d \theta .
$$

Equivalently,

$$
\begin{gathered}
\left(\frac{1}{2 \pi} \int_{0}^{2 \pi}|| D_{\alpha}[P]\left(e^{i \theta}\right)\left|+n m t\left(\frac{|\alpha|-1}{1+k^{\mu}}\right)\right|^{p} d \theta\right)^{1 / p} \\
\leq \frac{n\left(|\alpha|+k^{\mu}\right)}{\left(1 /(2 \pi) \int_{0}^{2 \pi}\left|k^{\mu}+e^{i \beta}\right| d \beta\right)^{1 / p}}\left(\frac{1}{2 \pi} \int_{0}^{2 \pi}\left|P\left(e^{i \theta}\right)\right|^{p} d \theta\right)^{1 / p},
\end{gathered}
$$

which immediately leads to (2.1) for $0<p<\infty$ and the cases $p=0$ and $p=\infty$ follow by respectively taking the limits $p \rightarrow 0^{+}$and $p \rightarrow \infty$. This completes the proof of Theorem 1 .

\section{Acknowledgement}

We are thankful to the referee for useful comments and suggestions.

\section{REFERENCES}

1. Arestov V. V. On integral inequalities for trigonometric polynomials and their derivatives. Math. USSRIzv., 1982. Vol. 18, No. 1. P. 1-17. DOI: 10.1070/IM1982v018n01ABEH001375

2. Arestov V. V. Integral inequalities for algebraic polynomials with a restriction on their zeros. Anal. Math., 1991. Vol. 17, P. 11-20. DOI: 10.1007/bf02055084 
3. Aziz A., Rather N. A. $L^{p}$ inequalities for polynomials. Glas. Math., 1997. Vol. 32, No. 1. P. $39-43$.

4. Aziz A., Rather N. A. Some Zygmund type $L^{q}$ inequalities for polynomials. J. Math. Anal. Appl., 2004. Vol. 289, No. 1. P. 14-29. DOI: 10.1016/S0022-247X(03)00530-4

5. Aziz A., Rather N.A. On an inequality concerning the polar derivative of a polynomial. Proc. Math. Sci., 2007. Vol. 117. P. 349-357. DOI: 10.1007/s12044-007-0030-0

6. Aziz A, Rather N.A., Aliya Q. $L_{q}$ norm inequalities for the polar derivative of a polynomial. Math. Inequal. Appl., 2008. Vol. 11. P. 283-296. DOI: 10.7153/mia-11-20

7. De Bruijn N G. Inequalities concerning polynomials in the complex domain. Indag. Math. (N.S.), 1947. Vol. 9, No. 5. P. 1265-1272.

8. Gardner R., Weems A. A Bernstein type $L^{p}$ inequality for a certain class of polynomials. J. Math. Anal. Appl., 1998. Vol. 219. P. 472-478.

9. Govil N. K. On the growth of polynomials. J. Inequal. Appl., 2002. Vol. 7, No. 5. P. 623-631.

10. Govil N. K., Rahman Q. I. Functions of exponential type not vanishing in a half-plane and related polynomials. Trans. Amer. Math. Soc., 1969. Vol. 137, P. 501-517. DOI: 10.1090/S0002-9947-1969-0236385-6

11. Mahler K. An application of Jensen's formula to polynomials. Mathematika, 1960. Vol. 7, No. 2. P. 98100. DOI: 10.1112/S0025579300001637

12. Marden M. Geometry of Polynomials. Math. Surveys, Amer. Math. Soc., 1989. 243 p.

13. Milovanovic G. V., Mitrinovic D.S., Rassias Th. Topics in Polynomials: Extremal properties, Inequalituies, Zeros. Singapore: World Scientific, 1994. 836 p. DOI: 10.1142/1284

14. Pólya G.,Szegö G. Aufgaben und lehrsätze aus der Analysis. Springer-Verlag, Berlin, 1925. 353 p. (in German)

15. Qazi M. A. On the maximum modulus of polynomials. Proc. Amer. Math. Soc., 1992. Vol. 115. P. 237243. DOI: 10.1090/S0002-9939-1992-1113648-1

16. Rahman Q. I., Schmeisser G. $L^{p}$ inequalities for polynomials. J. Approx. Theory, 1998. Vol. 53, No. 1. P. 26-32. DOI: 10.1016/0021-9045(88)90073-1

17. Rather N. A., Some integral inequalities for the polar derivative of a polynomial. Math. Balkanica (N.S.), 2008. Vol. 22, No. 3-4. P. 207-216.

18. Rather N. A. $L^{p}$ inequalities for the polar derivative of a polynomial. J. Inequal. Pure Appl. Math., 2008. Vol. 9, No. 4. Art. no. 103, P. 1-10.

19. Rather N. A., Iqbal A., Hyun G. H. Integral inequalities for the polar derivative of a polynomial. Nonlinear Funct. Anal. Appl., 2018. Vol. 23, No. 2. P. 381-393.

20. Schaeffer A. C. Inequalities of A. Markoff and S.Bernstein for polynomials and related functions. Bull. Amer. Math. Soc., 1941. Vol. 47. P. 565-579. DOI: 10.1090/S0002-9904-1941-07510-5

21. Zygmund A. A remark on conjugate series. Proc. Lond. Math. Soc. (3), 1932. Vol. s2-34, No. 1. P. 392400. DOI: $10.1112 / \mathrm{plms} / \mathrm{s} 2-34.1 .392$ 